\title{
Chromophore reduction plus reversible photobleaching: how the mKate2 "photoconversion" works
}

\author{
Elena A. Protasova ${ }^{1} \cdot$ Alexander S. Mishin ${ }^{2} \cdot$ Konstantin A. Lukyanov ${ }^{2} \cdot$ Eugene G. Maksimov $^{1}$. \\ Alexey M. Bogdanov²
}

Received: 2 March 2021 / Accepted: 25 May 2021 / Published online: 4 June 2021

(c) The Author(s), under exclusive licence to European Photochemistry Association, European Society for Photobiology 2021

\begin{abstract}
mKate red-to-green photoconversion is a non-canonical type of phototransformation in fluorescent proteins, with a poorly understood mechanism. We have hypothesized that the daughter mKate2 protein may also be photoconvertible, and that this phenomenon would be connected with mKate(2) chromophore photoreduction. Indeed, upon the intense irradiation of the protein sample supplemented by sodium dithionite, the accumulation of green as well as blue spectral forms is enhanced. The reaction was shown to be reversible upon the reductant's removal. However, an analysis of the fluorescence microscopy data, absorption spectra, kinetics and time-resolved fluorescence spectroscopy revealed that the short-wavelength spectral forms of mKate(2) exist before photoactivation, that their fractions increase light-independently after dithionite addition, and that the conversion is facilitated by the photobleaching of the red chromophore form.
\end{abstract}

Keywords Photoconversion $\cdot$ Fluorescent protein $\cdot$ Photobleaching $\cdot$ FRET $\cdot$ Chromophore $\cdot$ Dithionite

\section{Introduction}

The green fluorescent protein (GFP) from jellyfish Aequorea victoria, as well as its mutants and homologs, is widely used in the life sciences as a genetically encoded fluorescent label [1-3]. In the last two decades, many fluorescent proteins (FPs) with different spectral properties have been identified as the natural pigments in diverse marine creatures, including jellyfish $[4,5]$, corals and similar anthozoans [6-9], crustaceans [10], and even chordates [11]. In turn, hundreds of genetically engineered FP variants have been formulated from the natural proteins, thus greatly extending the available palette of fluorescence colors and providing new potential applications for FPs [12] (https://www.

Pushing the limits of flash photolysis to unravel the secrets of biological electron andproton transfer - a topical issue in honour of Klaus Brettel.

Alexey M. Bogdanov

noobissat@ya.ru

1 Faculty of Biology, Lomonosov Moscow State University, 119992 Moscow, Russia

2 Shemyakin-Ovchinnikov Institute of Bioorganic Chemistry, 117997 Moscow, Russia fpbase.org/table/). Photoactivatable (or phototransformable) FPs are essential parts of the fluorescence probe toolkit [13, 14]. In general, phototransformation involves a well-marked change in fluorophore behavior upon irradiation with light of a specific wavelength. In the case of FPs, such changes can involve the reversible switching between dark (off) and bright (on) states [15-17], the irreversible conversion to a spectral state with a distinct fluorescence emission color $[18,19]$, fluorescence kindling $[20,21]$, or combinations of reversible and irreversible transformations [22, 23]. Photoactivation increases the functionality of FPs as the tags used in advanced techniques for tracking macromolecules and subcellular structures, the mapping of intracellular trafficking, and signaling $[13,14]$. The single molecule localization microscopy (SMLM) approach [24, 25], wherein multiple repetitions of photoswitching cycles (switching the fluorescence on/off) within the probe subpopulation facilitate the further reconstruction of a fluorescence image with subdiffraction resolution, has given new significance to photoactivatable FPs [26, 27].

The currently known list of photoactivatable FPs is rather narrow compared to the comprehensive catalog of FPs. The molecular mechanisms underlying photoactivations are mostly well understood and imply intramolecular photochemistry (both covalent and non-covalent) with the 
involvement of the chromophore and its environment [14, 28]. FPs' irreversible photoconversions, based on intermolecular redox photoreactions, have also been documented [29-31]. The photoactivation ability is more widespread among FPs than is commonly believed [28]. Indeed, new photoactivation cases are continuously being reported [17, 23, 32], and their mechanisms often remain poorly understood. Inter alia, Kremers et al. described several unusual photoconversions, including the red-to-green conversion of the mKate and Katushka proteins observed both in cellulo (live HeLa cells) and in vitro (droplets of the purified protein) upon violet $(405 \mathrm{~nm})$ or orange $(561 \mathrm{~nm})$ laser irradiation [33]. While the authors focused on the photoconversion, with the molecular mechanism being beyond the paper's scope, they made remarkable discoveries that might point to the nonconventional nature of this phenomenon. Particularly, the authors observed time lags in both the reduction in red fluorescence and the increase in green fluorescence, detected the supralinear dependence of the conversion rate on the irradiation power, and reported the multiphasic character of increases in photoconverted species [33]. These features could indicate a complex photochemistry, including lightinduced redox reactions. Importantly, a green-emitting spectral form, structurally represented as an anionic GFP-type chromophore, is among the typical intermediates of the red FPs' chromophore maturation $[34,35]$. This form can be either a maturation dead-end product [34] or a direct precursor of the mature chromophore (at least in the proteins with an Asp-Tyr-Gly chromophore-forming triad) [36-39], and in both cases, it is separated from the mature chromophore by one oxidation reaction. One can speculate that the red-togreen photocoversions of mKate and Katushka are associated with the photoreduction of their chromophores to a green (anionic GFP) state. In the original paper [33], the authors expressed a similar supposition, emphasizing that if photoconversion yields an immature intermediate, then the process would be reversible upon the immature intermediate's further maturation; however, no data on the conversion's reversibility were reported. Alternatively, this conversion may operate similarly to the "greening" of DsRed, whereby the protein molecules carrying a green intermediate of the chromophore maturation pre-exist in the DsRed tetramer, and their fluorescence, normally masked by Förster resonance energy transfer (FRET), is evident upon the photobleaching of the mature red chromophore [40].

In this paper, we seek to better understand the nature of the mKate red-to-green photoconversion, clarify whether this process occurs in mKate2, test our hypotheses about the photoreduction effect and reversibility of the conversion, and specify the conversion's spectral and kinetic features.

\section{Materials and methods}

\subsection{Protein expression and purification}

mKate and mKate 2 coding sequences were cloned into the pQE30 vector (Qiagen) with an N-terminal 6xHis tag, expressed in the E. coli XL1 Blue strain (Invitrogen) and purified using TALON metal-affinity resin (Clontech). For further fluorescence microscopy experiments, the spherical resin particles with the immobilized proteins were washed four times with a phosphate-buffered saline (PBS pH 7.4, Gibco), 1:20 $\mathrm{v} / \mathrm{v}$. For the spectroscopy experiments, the proteins were solubilized using $100 \mathrm{mM}$ imidazole $\mathrm{pH} 8$, and then desalted by ultrafiltration with Amicon® Ultra-0.5 $10 \mathrm{~K}$ (Merck) filters (the final buffer was PBS pH 7.4).

\subsection{Fluorescence microscopy}

For fluorescence microscopy, an AF6000 LX microscope (Leica Microsystems, Wetzlar, Germany) with a $63 \times 1.4 \mathrm{NA}$ oil objective, a Photometrics (Tucson, AZ) CoolSNAP HQ CCD camera, and a $120 \mathrm{~W}$ HXP short arc lamp (Osram, Munich, Germany) were used. To detect the red fluorescence signal, a TX2 filter cube (excitation BP560/40, emission BP645/75), with a lamp intensity setting of 1 , binning of $1 \times 1$, and exposure lengths of 5-50 ms, was used. To detect the green fluorescence signal, a GFP filter cube (excitation BP470/40; emission BP525/50), with a lamp intensity setting of 3 , binning of $1 \times 1$, and exposure lengths of $30-100 \mathrm{~ms}$, was used. To detect the blue fluorescence signal, a BFP filter cube (excitation BP390/20; emission BP460/60), with lamp intensity settings of $2-3$, binning of $1 \times 1$, and exposure lengths of 150-200 ms, was used. To bleach/photoactivate mKate(2), 5 or $10 \mathrm{~s}$ illuminations, with a TX2 (or BFP in the case of blue-to-red conversion) cube with a lamp intensity setting of 5 , were employed. The selected field of view was recorded in time-lapse mode, wherein the sequence of detection (greenGFP, blue-BFP, and red-TX2) and activation (TX2 or BFP) frames was repeated 15-200 times without delay. To monitor the fluorescence recovery or decay, a single-channel time-lapse with $0.5-30$ min between frames was performed. The fluorescence data were all background-subtracted and normalized to either the maximum value (to show kinetics only) or the reference signal value (e.g., green/blue signals were normalized to the initial red signal to show their relative values). PBS ( $\mathrm{pH}$ 7.4) was used as a basic medium for the preparation of TALON-immobilized fluorescent protein suspensions. All the chemicals added to the protein were freshly dissolved in PBS and $\mathrm{pH}$-neutralized prior to the experiments. For each condition, at least three independent experiments (with 2-3 imaging series in each experiment) were performed. The microscopy data were processed using Leica Application Suite X 3.7, and 
the selected quantifications were performed in Origin Pro 2015 (OriginLab Corporation, USA).

\subsection{Steady-state absorption and fluorescence spectroscopy}

The absorbance and fluorescence spectra (except those shown in Fig. 6 and Sect. 3.2) were recorded using a Cary 100 UV/VIS spectrophotometer and a Cary Eclipse fluorescence spectrophotometer (Agilent), respectively. In all cases, a protein solution in PBS ( $\mathrm{pH}$ 7.4) was used. To bleach/ photoactivate mKate 2 in aqueous solutions, a laser beam $(\lambda=532 \mathrm{~nm}, 50 \mathrm{~mW})$ emitted by a continuous-wave LCSDTL-316 laser (LASER-COMPACT Co. Ltd) was applied. We irradiated a protein solution in either a cuvette $(0.5 \mathrm{~mL})$ or a plastic tube (small drop of $\sim 15 \mu \mathrm{L}$ ). The steady-state spectroscopy data were processed and visualized using Origin Pro 2015 (OriginLab Corporation, USA).

\subsection{Absorption kinetics measurement}

To determine the time constants characteristic of the formation of blue (and green) forms of mKate 2 with selected concentrations of dithionite, we measured changes in the mKate 2 absorption spectra in the $200-700 \mathrm{~nm}$ range, at defined wavelengths, using a Maya2000 Pro spectrometer (Ocean Insight, USA) and a deuterium lamp (SLS204, Thorlabs, USA).

\subsection{Time-resolved fluorescence spectroscopy}

The spectrally resolved fluorescence decay data of mKate 2 were recorded by a single-photon counting (SPC) detector with an ultra-low dark count rate (HPM-100-07C, Becker \& Hickl, Germany), in the spectral range from 450 to $650 \mathrm{~nm}$ with $5 \mathrm{~nm}$ steps, adjusted by an ML-44 monochromator (Solar, Belarus). Fluorescence was excited at $400 \mathrm{~nm}$ (repetition rate $80 \mathrm{MHz}$, pulse width $150 \mathrm{fs}$, optical power $5 \mathrm{~mW}$ ) using the second harmonics (ASG-O, Avesta Project LTD., Moscow, Russia) of a femtosecond optical parametric oscillator (TOPOL-1050-C, Avesta Project LTD.) pumped by a $\mathrm{Yb}$ femtosecond laser (TEMA-150, Avesta Project LTD.). The emission signal was collected perpendicular to the excitation beam. The temperature of the sample was stabilized during the experiment at $25^{\circ} \mathrm{C}$ by a cuvette holder (Qpod 2e) with a magnetic stirrer (Quantum Northwest, USA). Dithionite at concentrations of $0.5,4$ and $20 \mathrm{mM}$ was added to the sample (protein in PBS pH 7.4), and measurement of the fluorescence was conducted after the saturation of the chromophore reduction process and the accumulation of the protein with an immature chromophore, in order to avoid changes in spectral characteristics during scanning.
The global analysis of spectrally resolved fluorescence decay was preformed using Glotaran Software 1.5.1 (Biophysics department of the Vrije Universiteit Amsterdam [41]). The post-processing and visualization of calculated data were performed using an Origin Pro 2015 (OriginLab Corporation, USA).

Attempts to photoconvert mKate2 were made through two experimental setups: First, the sample was illuminated by the second harmonics of a $\mathrm{Yb}$ femtosecond laser $(525 \mathrm{~nm}$, $1.5 \mathrm{~W}$ average power) in a cuvette for about $10 \mathrm{~min}$. The second attempt was made under a microscope using a combination of $405 \mathrm{~nm}(5 \mathrm{~mW})$ and $510 \mathrm{~nm}(10 \mathrm{~mW})$ laser pulses focused through a $60 \times 0.7$ NA lens LUC PLAN FL (Olympus, Japan). In both cases, the fluorescence intensity of mKate 2 over time was monitored via a single-photon counting regime using the FIFO mode of the SPCM software (Becker \& Hickl, Germany). Between the cases, no significant differences in the fluorescence intensity of mKate2 were observed.

\section{Results}

We first tried to induce photoconversion in vitro via the irradiation of the purified mKate and mKate2, immobilized on the spherical resin particles $(50-100 \mu \mathrm{m})$, with green light $\left(560 \pm 20 \mathrm{~nm}, \sim 2 \mathrm{~W} / \mathrm{cm}^{2}\right)$ within a wide-field microscope (for details, see Sect. 2.2). Since both proteins showed similar behaviors in the pilot experiments, we pursued a detailed study of the brighter mKate 2 only and show the data for mKate 2 only in the manuscript. The protein-binding beads exhibited intense red and dim but detectable green fluorescence prior to photoactivation, demonstrating the weak appearance of the green signal and the gradual photobleaching of the red form throughout the experiment's course (Fig. 1). Kremers et al. showed a pre-activation green signal among the mKate fluorescence images (see Fig. 1a in Ref. [33]). It took about 150-300 s of irradiation for the green signal's value to reach a plateau (Fig. 1b). Remarkably, in our experiment, the contrast between the maximum and initial green signal was similar ( twofold increase) to that in the original paper, wherein photoactivation was carried out in live cells [33]. After the green signal reached a plateau, the photobleaching of the red form continued without any conversion (Fig. 1b). We also found that upon mKate(2) irradiation, along with the expected green form, a blue emissive form was produced, with comparable $(\sim 1.5$-fold activation) efficiency (Fig. 1). The ratio between the fluorescence intensities of the red, green, and blue species, normalized to exposure settings, light source spectrum and CCD-sensor spectral sensitivity, can be estimated as 100:4:0.03 before and 100:47:0.3 —after activation. 

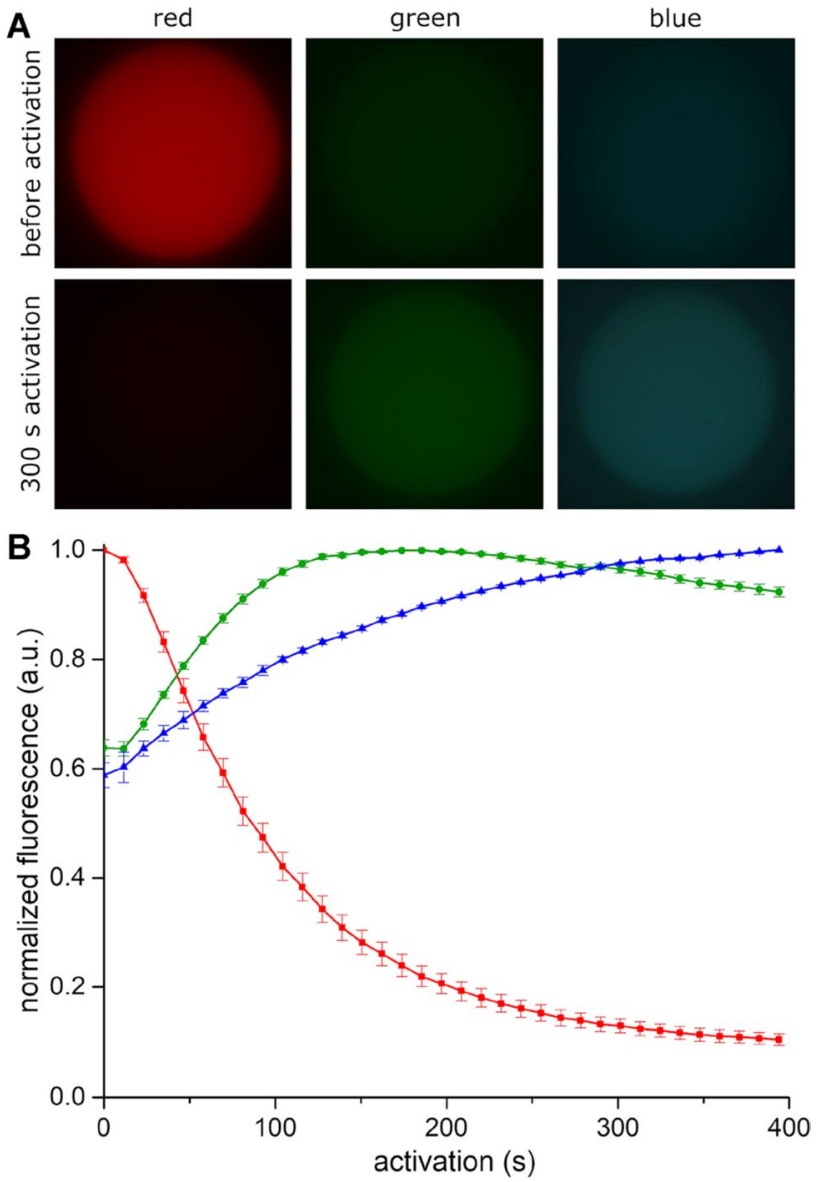

Fig. 1 Production of mKate2's green and blue fluorescent forms upon intense irradiation. a Wide-field fluorescence microscopy images (approximately $25 \%$ of the $63 \times$ objective field of view) recorded for three separate spectral channels before and after $300 \mathrm{~s}$ of illumination. b Kinetics of the red form's decay and short-wave species production. The data have been collected from the photoactivation $\left(560 \mathrm{~nm}, 2 \mathrm{~W} / \mathrm{cm}^{2}\right)$ time-lapse images by quantitative analysis of several regions of interest (ROIs). Fluorescence data were backgroundsubtracted and normalized to the maximum signal (error bars represent st.dev. values; $n=5$ )

In red fluorescent proteins (RFPs), blue emission usually originates from the neutral (protonated) GFP-type chromophore, which is the most common chromophore maturation intermediate in most RFPs with DsRed-type chromophores [34, 42]. Thus, several blue fluorescent proteins (e.g., TagBFP) were engineered by altering the chromophore maturation pathway, specifically, blocking it from developing past the neutral GFP state [43]. Taking into account previous studies on DsRed-type chromophore maturation [34], one can assume that both the blue and green fluorescent species forming upon mKate(2) photoconversion involve the previously described maturation intermediates, which undergo fewer oxidation steps than the mature red chromophore. To test this hypothesis, we assessed whether an external reducing agent affects the efficiency of the short-wave fluorescent species' production. To that end, we supplemented a suspension of mKate(2)-binding particles with sodium dithionite. This compound reverses green fluorescent protein chromophore maturation, specifically through a rate-limiting oxidation step [44]. Indeed, the addition of dithionite generally enhances mKate(2) conversion. Thus, the yield of the green form (rather, the plateau of the green signal's value) increased with $0.3-10 \mathrm{mM}$ of dithionite, though the conversion rate was found to be similar at all concentration points, including the control (see Fig. 2a). At low and medium concentrations, dithionite did not affect the photobleaching of the red form, but with $10 \mathrm{mM}$ of the reductant, it decayed around twofold faster than in the control (photobleaching half-times were $58 \pm 7 \mathrm{~s}$ at $0 \mathrm{mM}$ vs. $31 \pm 6 \mathrm{~s}$ at $10 \mathrm{mM}$; Fig. $2 \mathrm{~b}$ ). Notably, very little effect of the reducing agent's concentration on the blue form's production was found at concentrations less than $10 \mathrm{mM}$, but $10 \mathrm{mM}$ dithionite provided $\sim 50 \%$ increase in the yield of the blue form (Fig. 2c).

In most cases (except for dithionite concentrations $\geq 10 \mathrm{mM}$ ), at the beginning of the photoactivation timelapse series, we observed a time lag $(\sim 5 \mathrm{~s})$ in the appearance of the green form and the decay of the red form (similar behavior was reported in [33]); however, this was absent in the blue channel (Fig. 1, Suppl. Figure 1). The green form was characterized by moderate stability, decaying spontaneously at $\sim 1$ h half-time (Suppl. Figure 2A), whereas the blue one was stable (its intensity even appeared to be increasing). Importantly, we showed that dithionite-enhanced conversion is a completely reversible process. After the dithionite was washed out, we observed an $80-90 \%$ recovery of the red signal, which returned to the level detected before protein irradiation, accompanied by the disappearance of both green and blue forms (Fig. 2d, Suppl. Figure 2B). We also observed slight post-irradiation growth in green fluorescence ("dark phase" of the conversion), which is characteristic of the time-lapse series in which the green signal does not reach a plateau (see Suppl. Figure 3), and this effect was much more pronounced at high dithionite concentrations. Both of these observations suggest a chemical rather than photochemical phenomenon. Moreover, when we increased the dithionite concentration to $15-20 \mathrm{mM}$, an immediate light-independent conversion to the blue form was observed (Fig. 3). This process, which we hypothesized was a chemical reduction of the chromophore, was also found to be reversible. A similar observation was made with mCherry RFP, the chromophore of which can be reduced to a blueemitting form by beta-mercaptoethanol [45]. Using intense violet irradiation $\left(405 \pm 10 \mathrm{~nm}, 1 \mathrm{~W} / \mathrm{cm}^{2}\right)$, provided by a BFP filter cube, we succeeded in the partial photoconversion of the blue form back to the red state (Fig. 3). 

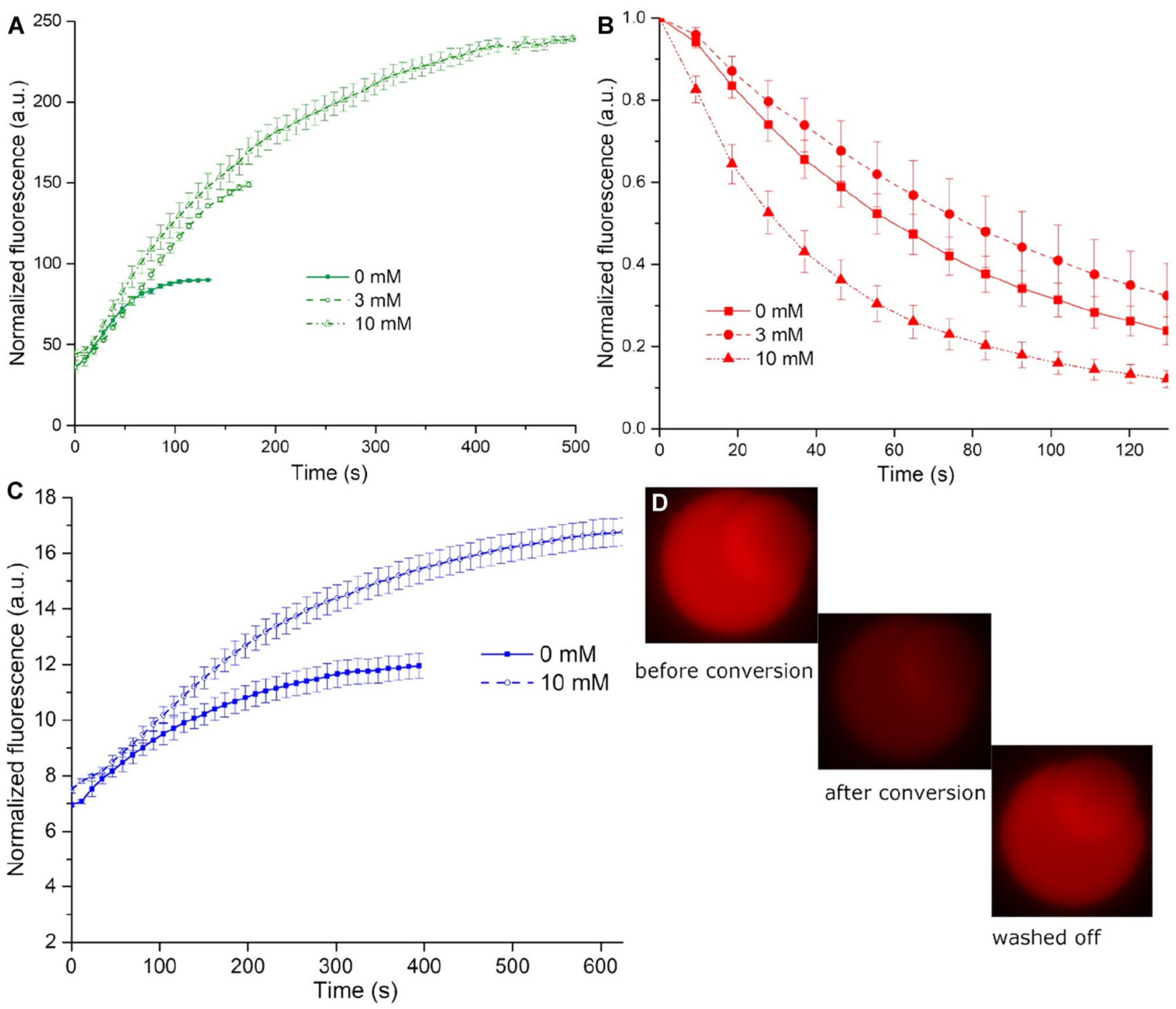

washed off

Fig. 2 Effect of dithionite and mKate2 emissive states' behavior on photoactivation. a Kinetics of the green fluorescence. As one can see, the rates of signal increase differ insignificantly, but the maximum signal plateau (yield) increases significantly upon dithionite addition. b The red form's photobleaching curves. An accelerated ( twofold) rate of decay with high dithionite content is observable. c Kinetics of the blue fluorescence. The weak relative effect of the reductant and the slightly different signal at the zero point are of note. d Example images showing the red form's recovery after protein pho-

We also tested the impacts of NADH $(1 \mathrm{mM})$, dithiothreitol (1-10 $\mathrm{mM})$, reduced glutathione $(5-40 \mathrm{mM})$ and hydroquinone (5-25 mM) on mKate2's conversion dynamics but derived no reliable results (data not shown). Among the chemicals that had some impact, beta-mercaptoethanol $(25 \mathrm{mM})$ slightly accelerated the photobleaching of the red form, with no effect on greening, while ascorbate $(25 \mathrm{mM})$ blocked the green form's appearance (Suppl. Figure 4). We tried to perform the photocoversion in a low-oxygen toactivation with $3 \mathrm{mM}$ dithionite. The upper-leftmost image was taken before intense irradiation, the central one after 1 min of illumination $\left(560 \mathrm{~nm}, 2 \mathrm{~W} / \mathrm{cm}^{2}\right)$, and the rightmost one immediately after the reductant was washed off. The fluorescence recovery was around $85 \%$. The data shown in (a), (b), and (c) represent the same field of view/ROI quantification. The fluorescence signals were backgroundsubtracted and normalized to the red signal at the zero point $(\mathbf{a}, \mathbf{c})$, or to the maximum signal (b), (error bars represent st.dev. values; $n=5$ )

environment (an argon-swept cell), but this had no effect (data not shown).

\subsection{Steady-state spectroscopy of the short-wave species}

We then commenced the steady-state spectroscopy measurements in the protein aqueous solution, focusing on mKate2's behavior. In mKate2's absorption spectrum, aside from the 


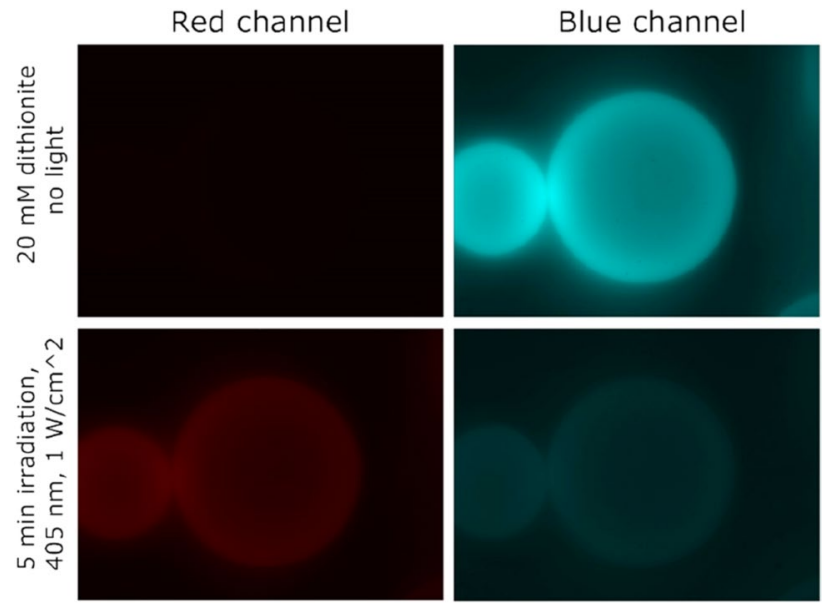

Fig. 3 mKate2's light-independent, dithionite-induced red-to-blue conversion (upper images), and its partial reversal (images on the bottom panel) through intense violet irradiation $\left(405 \mathrm{~nm}, 1 \mathrm{~W} / \mathrm{cm}^{2}\right)$

major peak of a mature chromophore (at $587 \mathrm{~nm}$ ), the broad band peaked at around $380 \mathrm{~nm}$ (Fig. 4a). This could be interpreted as the absorption of the immature chromophore fraction. Upon prolonged intense irradiation $(50 \mathrm{~mW}, 532 \mathrm{~nm}$ laser) of the protein sample without dithionite, only the photobleaching of the red form, not accompanied by qualitative spectrum changes, was detected (Fig. 4a). After the addition of dithionite (3-10 $\mathrm{mM})$, the main absorption peak's amplitude decreased, while the $400 \mathrm{~nm}$ band's absorption increased proportionally to the reductant's concentration, without irradiation (Fig. 4b). Upon the incremental irradiation of the protein solution supplemented with dithionite at medium concentrations (up to $5 \mathrm{mM}$ ), we detected a slight increase in the red peak's amplitude and a corresponding reduction in absorption in the violet region, both of which were dependent on the total irradiation time (Fig. 4c). This effect is likely connected to the kindling behavior of mKate2 (see [46], and Sect. 4), which dominates over the photobleaching at these irradiation power densities. Importantly, we observed no absorption increase (not to mention a peak) within the 450-500 $\mathrm{nm}$ region (where the green form was expected to absorb) at any concentration used (e.g., Fig. 4d). At high dithionite concentrations (>10 mM), the intense transition of the $587 \mathrm{~nm}$ absorption peak to a peak at $400 \mathrm{~nm}$ (reverse maturation), which was both post-irradiation (Fig. 4a) and spontaneous (Suppl. Figure 5), was recorded. After the dithionite was washed out (using ultrafiltration or dialysis), chromophore re-maturation (blue-to-red spontaneous conversion) occurred on a timescale of hours (Suppl. Figure 5). The absorption of the green form was not visible during the re-maturation.

In the steady-state fluorescence spectra of the non-photobleached mKate2, pre-existing green $\left(\lambda_{\mathrm{ex}} / \lambda_{\mathrm{em}}=484 / 508 \mathrm{~nm}\right)$ and blue $\left(\lambda_{\mathrm{ex}} / \lambda_{\mathrm{em}}=354 / 442 \mathrm{~nm}\right)$ emissive states were detected, along with the major red form $\left(\lambda_{\mathrm{ex}} / \lambda_{\mathrm{em}}=589 / 628 \mathrm{~nm}\right)$ (Fig. 5a). After the addition of dithionite ( $3 \mathrm{mM})$, the blue form's excitation maximum slightly increased its amplitude and underwent a pronounced spontaneous bathochromic shift to $380 \mathrm{~nm}$ (Fig. 5b). Remarkably, red fluorescence was also effectively excited at $380 \mathrm{~nm}$ in the presence of dithionite (Fig. 5b), probably indicating the FRET from the newly produced blue-emitting chromophore to a mature red one. Further increases in dithionite concentration (to $20 \mathrm{mM}$ ) resulted in a further increase in amplitude and a bathochromic shift in the excitation maximum $(\sim 405 \mathrm{~nm})$, while the emission spectrum recorded at this excitation showed a major peak at $460 \mathrm{~nm}$, indicating lightindependent chromophore reduction occurrence (Fig. 5b). Relative to the control, the contribution of the green form increased slightly with the medium dithionite concentration but dropped again with the high concentration (Suppl. Figure 6). Interestingly, the green form's emission band showed bimodality (peaks at $\sim 510$ and $\sim 525 \mathrm{~nm}$ ) in the absence of dithionite and at its medium concentrations, and it became single-peaked $(\sim 525 \mathrm{~nm})$ with the increase in dithionite concentration (Suppl. Figure 6).

The prolonged irradiation of the samples, both supplemented with dithionite at a medium concentration and used without the reductant, led mainly to the photobleaching of the red form (more clearly expressed in the presence of the reductant), with a barely visible change in the shape of the green emissive spectrum (Suppl. Figure 7). The green fluorescence of the sample irradiated in the presence of dithionite is excited mostly at $\sim 405 \mathrm{~nm}$, which reveals its relation to the blue-absorbing form and might indicate the origin of the green signal in FRET and/or ESPT (Fig. 5c).

We then monitored the absorption kinetics upon dithionite addition. Since we could not detect the green form's absorption, we focused on monitoring blue $(400 \mathrm{~nm})$ and red $(580 \mathrm{~nm})$ absorbance, trying to determine the time constants of their changes (Fig. 6).

At both the tested concentrations of sodium dithionite ( 1 and $20 \mathrm{mM}$ ), we observed very similar kinetics, with a decrease in the red peak and an increase in the blue peak (Fig. 6). The coincidence of the characteristic time constants of the red form of the chromophore decaying and the blue form appearing indicates the interconnection of these processes (i.e., red-to-blue chemical conversion).

\subsection{Time-resolved spectroscopy}

To explore the phototransformations of mKate 2 in more detail, we performed femtosecond time-resolved fluorescence spectroscopy measurements, wherein the monitoring of the properties and evolution of the spectral forms with high precision is possible. 

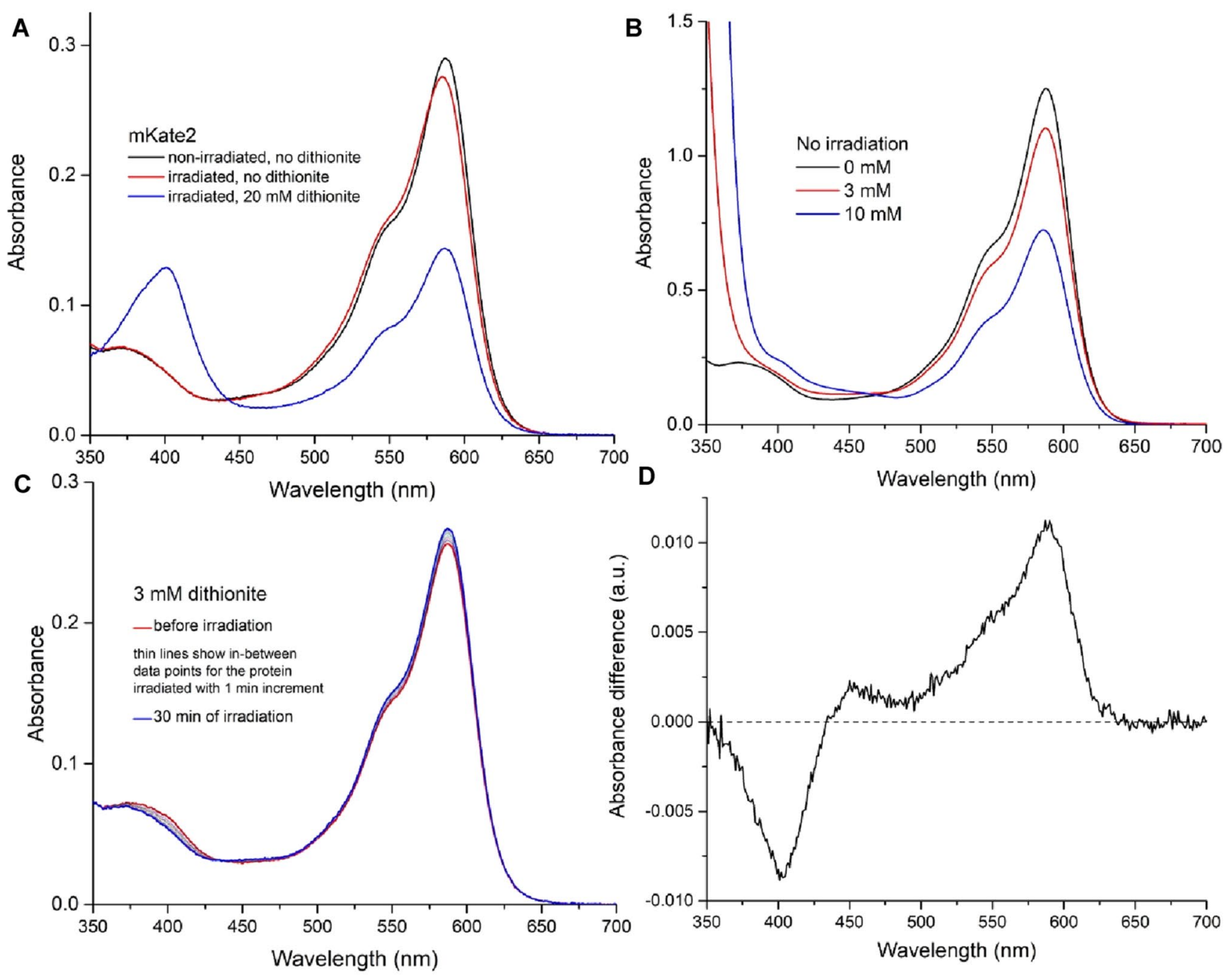

Fig. 4 Absorption spectra of the mKate 2 aqueous solution in the near-UV-visible range, demonstrating the influence of a prolonged irradiation $(50 \mathrm{~mW}, 532 \mathrm{~nm}, 15 \mathrm{~min}$ in $15 \mu \mathrm{l})$ and irradiation + high dithionite concentration; $\mathbf{b}$ different dithionite concentrations without irradiation; $\mathbf{c}$ an incremental irradiation $(50 \mathrm{~mW}, 532 \mathrm{~nm}$ in $0.5 \mathrm{~mL}$ ) at a medium dithionite concentration. In the latter case, the thick red line depicts absorption before illumination, the thick blue line depicts absorption after 30 min of illumination, and the thin lines show the intermediate spectra recorded with 1-min increments. d Difference absorption spectrum based on the outermost spectra shown in (c). Upon photoactivation, the $400 \mathrm{~nm}$-absorbing form is consumed along with the increase in the $580 \mathrm{~nm}$-absorbing form. No significant changes are observable within the $450-500 \mathrm{~nm}$ region
We recorded the spectrally resolved fluorescence decay of mKate 2 by performing fluorescence excitation at $405 \mathrm{~nm}$ with a femtosecond laser and detected the signal with a single-photon counting device (see Sect. 2.5, Suppl. Figure 8). The global analysis (Glotaran Software, https://glota ran.org/) of these data has revealed three components with lifetimes in the $100 \mathrm{ps}-3 \mathrm{~ns}$ range. The $2.4-\mathrm{ns}$ component is the red "mature" chromophore (Fig. 7). Although the emission of the red state dominates the steady-state spectrum of mKate2, with $405 \mathrm{~nm}$ excitation, its concentration is about three times lower than the green state. This is presumably due to the differences in the values of molar extinction at $405 \mathrm{~nm}$ for the red and green states.

Upon the addition of dithionite and with increases in its concentration, a reduction in the fluorescence intensity of the red state was observed, while its lifetime was constant. The reduction in average lifetime in the $600-650 \mathrm{~nm}$ region is associated with the increasing contribution of the blue and green states. The fluorescence decay of blue states involves two components with subnanosecond lifetimes, the duration of which increased by approximately half in the presence of dithionite (Fig. 7a). While the green and red states are still visible with $0.5 \mathrm{mM}$ of dithionite, at 

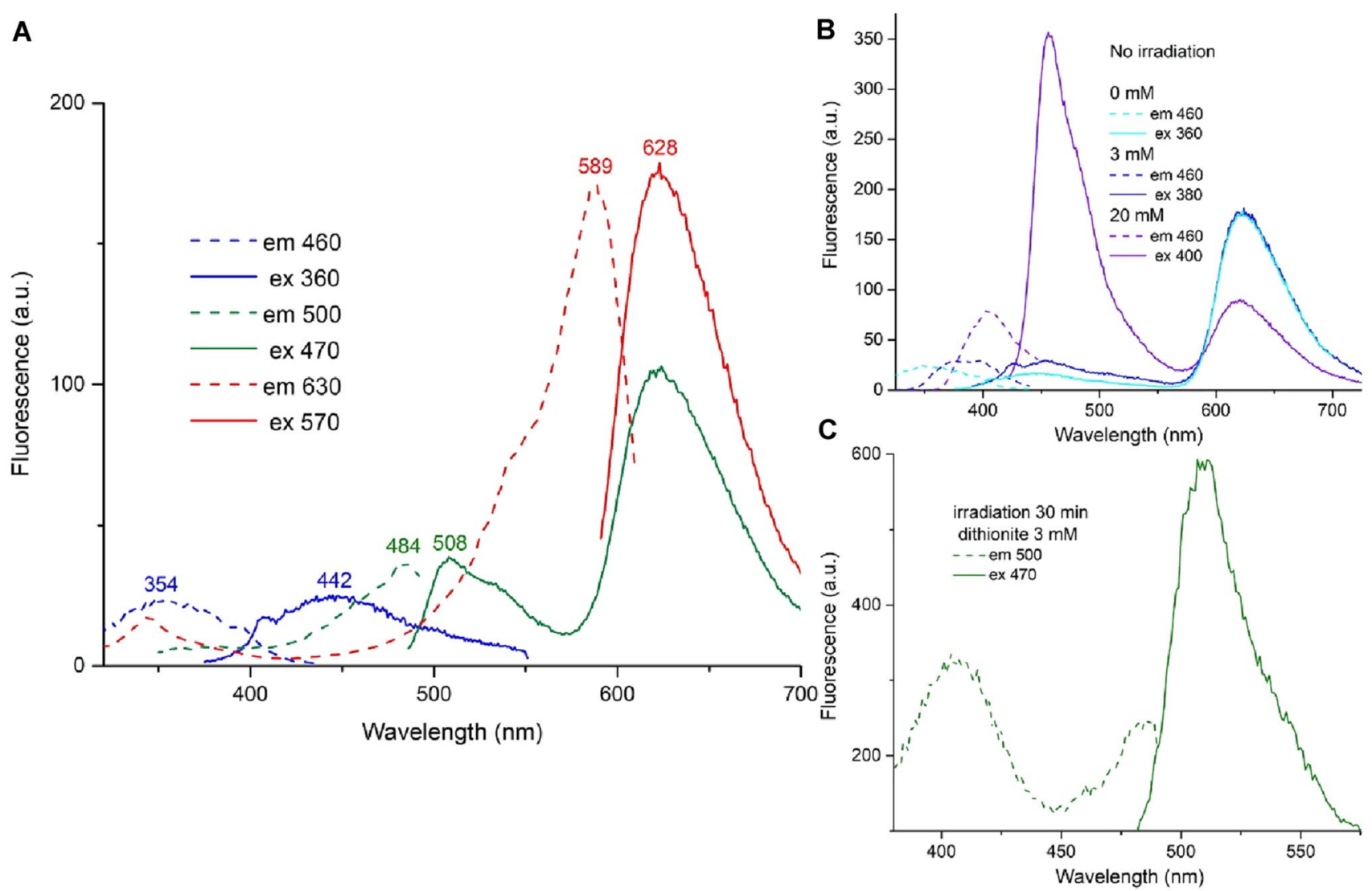

Fig. 5 Steady-state fluorescence spectra of the mKate2 aqueous solution. a Fluorescence excitation (dashed lines) and emission (solid lines) spectra of the blue (blue lines), green (green lines), and red (red lines) spectral species pre-existing in the non-irradiated PBS solution of mKate2. b Fluorescence excitation and emission spectra of the blue form appearing in the non-irradiated dithionite-supplemented $(0 \mathrm{mM}$-in cyan, $3 \mathrm{mM}$ in blue, $20 \mathrm{mM}$ in violet) $\mathrm{mKate} 2$

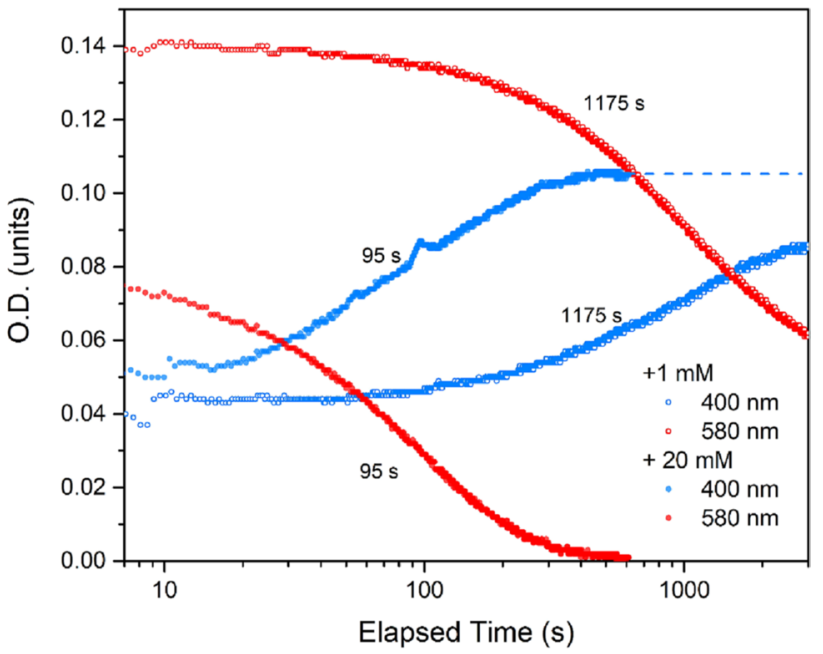

Fig. 6 Time courses of optical density (O.D.) at $400 \mathrm{~nm}$ (blue dots) and $580 \mathrm{~nm}$ (red dots) after addition of 1 or $20 \mathrm{mM}$ of dithionite to the solution of mKate2. Measurements were conducted at $25{ }^{\circ} \mathrm{C}$ with constant stirring solution. The red emission peaks excited at $\sim 400 \mathrm{~nm}$ are also visible. The spectra of the three concentrations were recorded using the same instrument settings. $\mathbf{c}$ Fluorescence spectra of the green form appearing in the irradiated $(50 \mathrm{~mW}, 532 \mathrm{~nm}$ in $0.5 \mathrm{~mL}$ ) and dithionitesupplemented $(3 \mathrm{mM})$ mKate 2 solution. The major excitation mode at $400 \mathrm{~nm}$ is worth noting. In all cases, the non-normalized (to excitation intensity/gain) data are shown

higher concentrations, almost the whole fluorescence signal is given by the blue state of the chromophore (Fig. 7).

Assuming that the molar extinction of the red state (and other states) is constant (not affected by dithionite), the relative reduction in the concentration of this state in the presence of $20 \mathrm{mM}$ dithionite is about $97 \%$. Simultaneously, the concentration of the blue state (peaking at $460 \mathrm{~nm}$ ) increases by about 50 -fold, which indicates that not only red states, but also green states, are converted into blue states.

No significant impact of light irradiation (photoactivation) on the fluorescence kinetics was observed. We irradiated the protein solution with both a green laser in a cuvette $(525 \mathrm{~nm}, 1.5 \mathrm{~W}$ average power) and a violet laser under a microscope $(405 \mathrm{~nm}, 5 \mathrm{~mW}$, with a $60 \times 0.7 \mathrm{NA}$ objective; see Sect. 2.5). In both cases, the fluorescence intensity kinetics of mKate 2 were monitored with a singlephoton counting regime, and no significant changes were observed. 


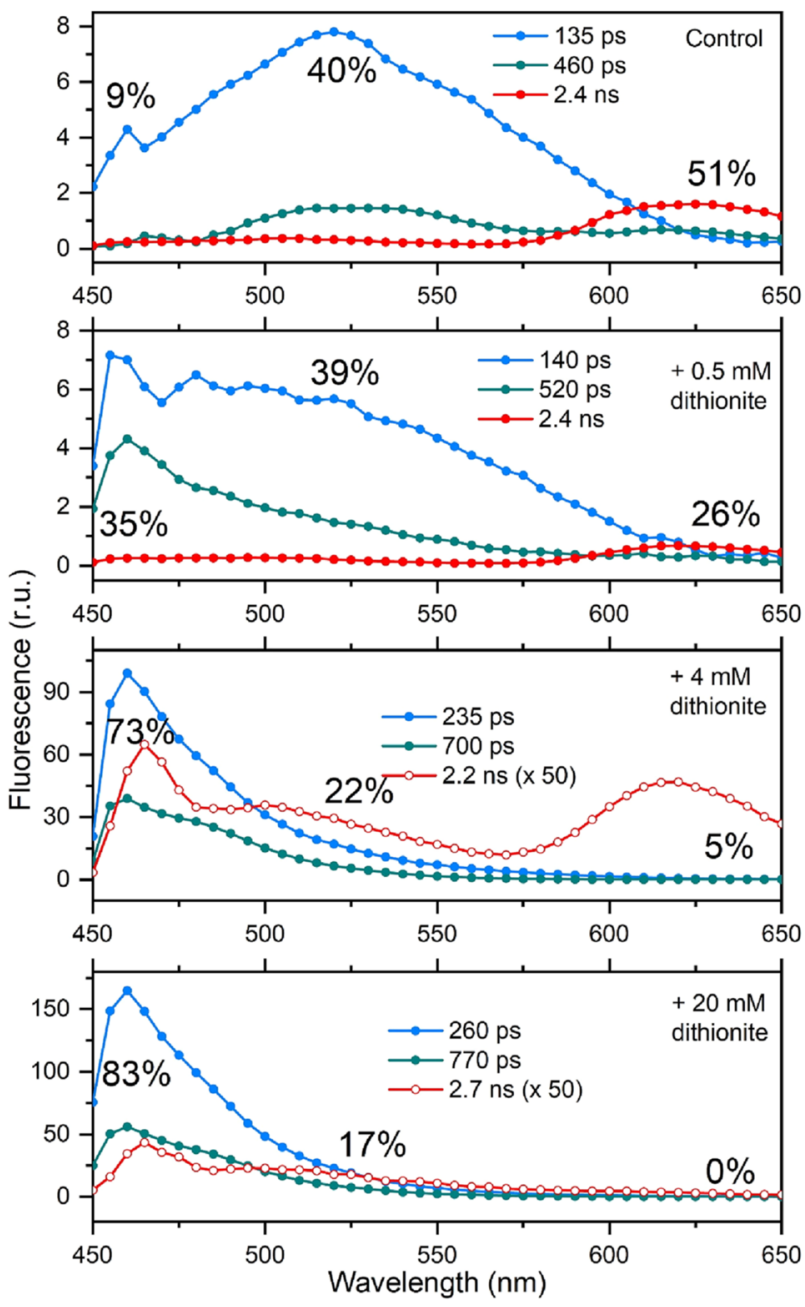

Fig. 7 Decay-associated spectra of mKate 2 excited at $405 \mathrm{~nm}$ in the absence and presence of increasing concentrations of dithionite. An additional component ( $30 \mathrm{ps})$ was detected via global analysis of the data at $470 \mathrm{~nm}$, which is responsible for the Raman scattering of water (data not shown). Note that at 4 and $20 \mathrm{mM}$, the amplitude of the 2-ns component (red line) was multiplied by 50 for clearer presentation. Relative contribution of photons detected within the different spectral regions $(460,510$ and $630 \mathrm{~nm})$ are indicated above the curves

Finally, we examined the photobehavior of mKate2, immobilized on the resin particles, with the fluorescence lifetime imaging microscopy (FLIM). To that end, we acquired the fluorescence decay data within the three spectral regions $(460,510$ and $630 \mathrm{~nm})$ immediately after laser switch-on and $60 \mathrm{~s}$ post the sample irradiation (Fig. 8).

While the lifetimes of both blue and green emissive species decreased after irradiation, indicating reduction in their fluorescence quantum yields, the red form of nonirradiated protein showed a 40-ps delay in fluorescence decay compared to irradiated one. We attribute this time gap to excitation energy transfer from the short-wave emitting species.

\section{Discussion and conclusion}

As was the case with mKate [33], in mKate2, a small fraction of the green emissive state can be found in the protein sample prior to irradiation, independently of the presence of dithionite. We also identified the blue form, which is also present in the non-activated protein and, unlike the green one, can be generated light-independently when the protein interacts with the reducing agent. Importantly, we detected no signatures suggesting the accumulation of the green form $\left(\lambda_{\mathrm{abs}} \sim 450-500 \mathrm{~nm}\right)$ under any experimental conditions in any of the absorption measurements. As such, the green fluorescence intensity cannot be considered a reliable indicator of photoconversion, because its manifestation depends not only on the amount of green chromophore, but also on the FRET (or even a radiative energy transfer; see below) efficiency from the blue to green form (wherein the green form acts as an energy acceptor), as well as from the green to the red form (where the green form is the donor). Accordingly, an increase in the green fluorescence intensity is expected when the red form is degraded (for example, during photobleaching) and when the amount of the blue form that is present increases. We observed both these effects in the microscopy experiments. Due to the extremely high protein concentration on the surface of the metal-affinity resin, we must consider local effects on the optical properties of mKate(2). Considering the size of the resin (diameter $\sim 100 \mu \mathrm{m}$ ) and the typical number of NTAs, around 1 billion protein molecules could be bound to a single particle. This means that the local concentration of the protein could be about $1.2 \mathrm{mM}$, and the distance between two molecules could be about $10 \mathrm{~nm}$. This might lead to effects similar to the oligomerization of the protein, resulting in the reabsorption of fluorescence and (all possible types of) FRET. The impact of the dithionite is twofold here: on the one hand, it promotes the accelerated photobleaching of the red form (Fig. 2b) and some light-independent reductions in its concentration (Fig. 4b); on the other hand, it facilitates the lightindependent production of the blue form (Figs. 3, 4a, 5b, 6, 7 ). The fact that we observed only a weak enhancement of the blue signal in the presence of dithionite via fluorescence microscopy (which contrasts the efficient blue form production observed via absorption spectroscopy) also indirectly proves the role of FRET in the green signal's enrichment. Moreover, since the structure of the green and blue emissive forms is most probably a GFP-type chromophore (in the anionic and neutral states, respectively), one cannot exclude the effect of excited-state proton transfer (ESPT [47]) on their fluorescence behavior, namely green emission under violet excitation. The manifestation of green fluorescence seems to show a complex dependence on the behavior of two other emissive states, which explains the multiphasic character 

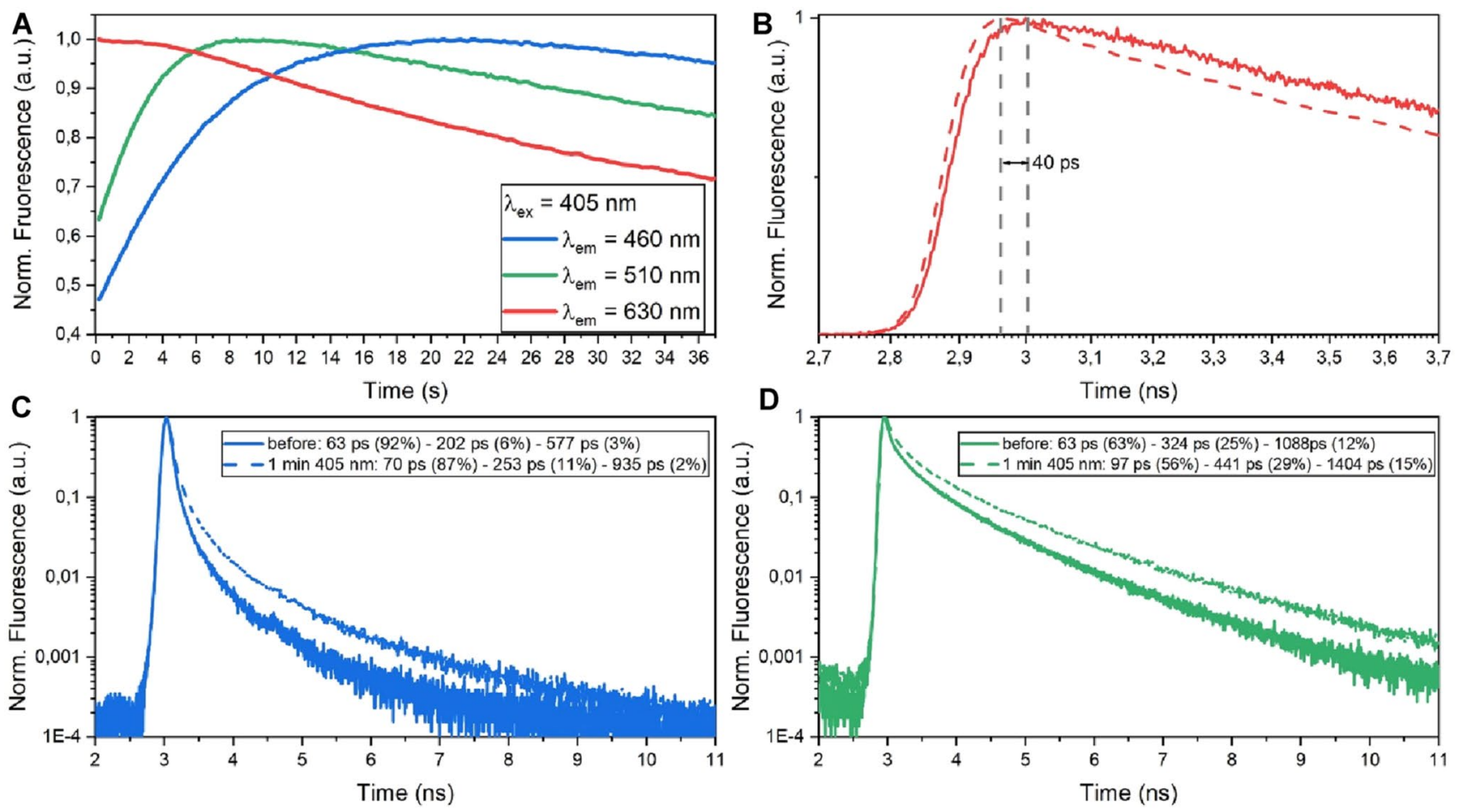

Fig. 8 Fluorescence emission of the immobilized mKate2 recorded via (a) intensity monitoring (FIFO mode) upon the continuous excitation with a $405 \mathrm{~nm}$ laser $(250 \mu \mathrm{W}, 60 \times$ objective $)$ within the blue, green and red spectral regions; fluorescence decay measurement started immediately after laser switch-on (solid lines) and $60 \mathrm{~s}$ post

and the supralinear power-dependence of the green signal's appearance, as reported by Kremers et al. [33].

However, the role of FRET in the observed interrelations between mKate(2)'s emissive states may be questioned due to the nominal monomericity of these proteins [48, 49]. Indeed, while DsRed greening [40] associated with protein molecules that have an immature chromophore within the RFP tetramer seems like a logically sound phenomenon, similar behavior in monomeric RFPs seems almost impossible. However, it is difficult to define mKate and mKate 2 as strict monomers, especially at concentrations characteristic of their overexpression in live cells [50], in concentrated aqueous solutions [51] and on protein-binding particles. We, therefore, believe that even a small fraction of the mKate(2) dimers and/or tetramers containing protein molecules with an immature chromophore would be sufficient to facilitate greening.

A time lag in the photobleaching and the appearance of the green form provides further evidence of the FRET relationship between mKate(2)'s red and green fluorescence. This time lag is associated with mKate [49] and mKate2's [46] fluorescence kindling behavior, described earlier. Interestingly, in our experiments, this phenomenon differed depending on the irradiation power densities, from

the sample irradiation (dashed lines) within (b) the red, (c) the blue and (d) the green spectral regions. A 40 ps time delay between decay curves of the red emissive species is indicated by the dashed vertical lines. Exponential fitting results are shown in the boxes inside the panels

non-observable or weak photoactivation (at $\sim$ milliWatts/ $\mathrm{cm}^{2}$, Fig. $4 \mathrm{c}$ ) to the aforementioned photobleaching time lag (at $\sim$ Watts $/ \mathrm{cm}^{2}$, Suppl. Figure 1). Photobleaching is the last key aspect of this phenomenon. The partial reversibility of the red form's decay, as observed after the activation, could be attributed to its mixed photobleaching mechanism, which combines both reversible and permanent components. RFPs generally undergo reversible transitions to dark states [52-54], which is part of the photobleaching that takes place during fluorescent imaging (these are usually visible at the early phase of the probe photobleaching). Thus, while the reversibility of the chemical red-to-blue conversion after the reductant is washed off could be attributed to the chromophore's re-maturation, the reversibility observed after the dithionite-free "photoconversion" is most likely associated with the red form returning to a bright state. Regardless, dithionite accelerates the bleaching of the red form, and this, along with the production of the blue form, is an important factor in the enhancement of greening (at least at $\geq 5 \mathrm{mM}$ dithionite). To combine our observations and assumptions within a unified model, we present a schematic representing (photo) behavior of mKate(2) under different conditions (Suppl. Figure 9). 
It seems that some interesting details of this process remain to be further studied. We have observed signs of heterogeneity within the green and blue forms' spectral identities, particularly the green state emission spectrum's bimodality (Suppl. Figure 6), the two lifetime components of the blue state's fluorescence decay (Fig. 7 and Suppl. Figure 8), and the bathochromic shift of the blue form's absorption/excitation (Figs. 4a and 5b). Here, we have not provided an explanation for these phenomena, but we believe that their further study might help in deciphering the peculiarities of the RFP chromophores' maturation, for example, via the transient absorption method.

To conclude, mKate(2)'s photoconversion (which, strictly speaking, is not a photoconversion) was more puzzling than expected. However, as we have shown, the molecular mechanism of this phenomenon is rather trivial and, unfortunately, not related to the photoinduced chromophore reduction.

Supplementary Information The online version contains supplementary material available at https://doi.org/10.1007/s43630-021-00060-8.

Acknowledgements This study was supported by the Russian Science Foundation (RSCF), Grant № 20-14-00255. The funders of the study had no part in the writing, analysis, interpretation, or decision to submit the paper. Experiments were partially carried out using equipment provided by the IBCH core facility (CKP IBCH, supported by Russian Ministry of Education and Science, Grant RFMEFI62117X0018).

Author contributions EAP: investigation, formal analysis, software; ASM: validation, investigation; KAL: conceptualization, supervision, resources; EGM: methodology, validation, formal analysis, investigation, visualization; AMB: conceptualization, investigation, project administration, funding acquisition. The manuscript was written through contributions of all authors. All authors have given approval to the final version of the manuscript.

Funding This study was supported by the Russian Science Foundation (RSCF), Grant № 20-14-00255.

Availability of data and material Supplementary information is available along with an online-version of the manuscript at https://doi.org/ 10.1007/s43630-021-00060-8. The datasets generated during and/or analyzed during the current study are available from the corresponding author on reasonable request.

Code availability Not applicable.

\section{Declarations}

Conflict of interest There are no conflicts of interest.

Ethics approval Not applicable.

Consent to participate Not applicable.

Consent for publication Not applicable.

\section{References}

1. Chudakov, D. M., Matz, M. V., Lukyanov, S., \& Lukyanov, K. A. (2010). Fluorescent proteins and their applications in imaging living cells and tissues. Physiological Reviews, 90(3), 1103-1163. https://doi.org/10.1152/physrev.00038.2009

2. Mishin, A. S., Belousov, V. V., Solntsev, K. M., \& Lukyanov, K. A. (2015). Novel uses of fluorescent proteins. Current Opinion in Chemical Biology, 27, 1-9. https://doi.org/10.1016/j.cbpa.2015. 05.002

3. Cardarelli, F. (2020). Back to the future: Genetically encoded fluorescent proteins as inert tracers of the intracellular environment. International Journal of Molecular Sciences. https://doi.org/10. 3390/ijms 21114164

4. Aglyamova, G. V., Hunt, M. E., Modi, C. K., \& Matz, M. V. (2011). Multi-colored homologs of the green fluorescent protein from hydromedusa Obelia sp. Photochemical \& Photobiological Sciences: Official journal of the European Photochemistry Association and the European Society for Photobiology, 10(8), 1303-1309. https://doi.org/10.1039/c1pp05068k

5. Lambert, G. G., Depernet, H., Gotthard, G., Schultz, D. T., Navizet, I., Lambert, T., \& Shaner, N. C. (2020). Aequorea's secrets revealed: New fluorescent proteins with unique properties for bioimaging and biosensing. PLoS Biology, 18(11), e3000936. https://doi.org/10.1371/journal.pbio.3000936

6. Matz, M. V., Fradkov, A. F., Labas, Y. A., Savitsky, A. P., Zaraisky, A. G., Markelov, M. L., \& Lukyanov, S. A. (1999). Fluorescent proteins from nonbioluminescent Anthozoa species. Nature biotechnology, 17(10), 969-973. https://doi.org/10.1038/ 13657

7. Salih, A., Larkum, A., Cox, G., Kühl, M., \& Hoegh-Guldberg, O. (2000). Fluorescent pigments in corals are photoprotective. Nature, 408(6814), 850-853. https://doi.org/10.1038/35048564

8. Wiedenmann, J., Schenk, A., Röcker, C., Girod, A., Spindler, K.-D., \& Nienhaus, G. U. (2002). A far-red fluorescent protein with fast maturation and reduced oligomerization tendency from Entacmaea quadricolor (Anthozoa, Actinaria). Proceedings of the National Academy of Sciences of the United States of America, 99(18), 11646-11651. https://doi.org/10.1073/pnas. 182157199

9. Karasawa, S., Araki, T., Nagai, T., Mizuno, H., \& Miyawaki, A. (2004). Cyan-emitting and orange-emitting fluorescent proteins as a donor/acceptor pair for fluorescence resonance energy transfer. The Biochemical journal, 381(Pt 1), 307-312. https://doi.org/10. 1042/BJ20040321

10. Shagin, D. A., Barsova, E. V., Yanushevich, Y. G., Fradkov, A. F., Lukyanov, K. A., Labas, Y. A., \& Matz, M. V. (2004). GFPlike proteins as ubiquitous metazoan superfamily: Evolution of functional features and structural complexity. Molecular Biology and Evolution, 21(5), 841-850. https://doi.org/10.1093/molbev/ msh079

11. Deheyn, D. D., Kubokawa, K., McCarthy, J. K., Murakami, A., Porrachia, M., Rouse, G. W., \& Holland, N. D. (2007). Endogenous green fluorescent protein (GFP) in amphioxus. The Biological Bulletin, 213(2), 95-100. https://doi.org/10.2307/25066625

12. Lambert, T. J. (2019). FPbase: A community-editable fluorescent protein database. Nature Methods, 16(4), 277-278. https://doi.org/ 10.1038/s41592-019-0352-8

13. Lukyanov, K. A., Chudakov, D. M., Lukyanov, S., \& Verkhusha, V. V. (2005). Innovation: Photoactivatable fluorescent proteins. Nature Reviews. Molecular Cell Biology, 6(11), 885-891. https:// doi.org/10.1038/nrm1741

14. Adam, V., Berardozzi, R., Byrdin, M., \& Bourgeois, D. (2014). Phototransformable fluorescent proteins: Future challenges. 
Current Opinion in Chemical Biology, 20, 92-102. https://doi. org/10.1016/j.cbpa.2014.05.016

15. Chang, H., Zhang, M., Ji, W., Chen, J., Zhang, Y., Liu, B., \& $\mathrm{Xu}, \mathrm{T}$. (2012). A unique series of reversibly switchable fluorescent proteins with beneficial properties for various applications. Proceedings of the National Academy of Sciences of the United States of America, 109(12), 4455-4460. https://doi.org/10.1073/ pnas. 1113770109

16. Jablonski, A. E., Vegh, R. B., Hsiang, J.-C., Bommarius, B., Chen, Y.-C., Solntsev, K. M., \& Dickson, R. M. (2013). Optically modulatable blue fluorescent proteins. Journal of the American Chemical Society, 135(44), 16410-16417. https://doi.org/10.1021/ja405 $459 \mathrm{~b}$

17. Pennacchietti, F., Serebrovskaya, E. O., Faro, A. R., Shemyakina, I. I., Bozhanova, N. G., Kotlobay, A. A., \& Testa, I. (2018). Fast reversibly photoswitching red fluorescent proteins for live-cell RESOLFT nanoscopy. Nature Methods, 15(8), 601-604. https:// doi.org/10.1038/s41592-018-0052-9

18. Chudakov, D. M., Verkhusha, V. V., Staroverov, D. B., Souslova, E. A., Lukyanov, S., \& Lukyanov, K. A. (2004). Photoswitchable cyan fluorescent protein for protein tracking. Nature Biotechnology, 22(11), 1435-1439. https://doi.org/10.1038/nbt1025

19. Zhang, M., Chang, H., Zhang, Y., Yu, J., Wu, L., Ji, W., \& Xu, T. (2012). Rational design of true monomeric and bright photoactivatable fluorescent proteins. Nature Methods, 9(7), 727-729. https://doi.org/10.1038/nmeth.2021

20. Patterson, G. H., \& Lippincott-Schwartz, J. (2002). A photoactivatable GFP for selective photolabeling of proteins and cells. Science, (New York, N.Y.), 297(5588), 1873-1877. https://doi. org/10.1126/science. 1074952

21. Chudakov, D. M., Belousov, V. V., Zaraisky, A. G., Novoselov, V. V., Staroverov, D. B., Zorov, D. B., \& Lukyanov, K. A. (2003). Kindling fluorescent proteins for precise in vivo photolabeling. (pp. 191-194). United States. https://doi.org/10.1038/nbt778

22. Adam, V., Moeyaert, B., David, C. C., Mizuno, H., Lelimousin, M., Dedecker, P., \& Hofkens, J. (2011). Rational design of photoconvertible and biphotochromic fluorescent proteins for advanced microscopy applications. Chemistry \& Biology, 18(10), 12411251. https://doi.org/10.1016/j.chembiol.2011.08.007

23. Solovyev, I. D., Gavshina, A. V., \& Savitsky, A. P. (2019). Novel phototransformable fluorescent protein SAASoti with unique photochemical properties. International Journal of Molecular Sciences, 20(14), 3399. https://doi.org/10.3390/ijms20143399

24. Betzig, E., Patterson, G. H., Sougrat, R., Lindwasser, O. W., Olenych, S., Bonifacino, J. S., \& Hess, H. F. (2006). Imaging intracellular fluorescent proteins at nanometer resolution. Science (New York, N.Y.), 313(5793), 1642-1645. https://doi.org/10.1126/scien ce. 1127344

25. Rust, M. J., Bates, M., \& Zhuang, X. (2006). Sub-diffractionlimit imaging by stochastic optical reconstruction microscopy (STORM). Nature Methods, 3(10), 793-795. https://doi.org/10. 1038/nmeth929

26. Nienhaus, K., \& Nienhaus, G. U. (2014). Fluorescent proteins for live-cell imaging with super-resolution. Chemical Society Reviews, 43(4), 1088-1106. https://doi.org/10.1039/c3cs60171d

27. Li, H., \& Vaughan, J. C. (2018). Switchable fluorophores for single-molecule localization microscopy. Chemical Reviews, 118(18), 9412-9454. https://doi.org/10.1021/acs.chemrev.7b007 67

28. Acharya, A., Bogdanov, A. M., Grigorenko, B. L., Bravaya, K. B., Nemukhin, A. V., Lukyanov, K. A., \& Krylov, A. I. (2017). Photoinduced chemistry in fluorescent proteins: Curse or blessing? Chemical Reviews, 117(2), 758-795. https://doi.org/10.1021/ acs.chemrev.6b00238

29. Bogdanov, A. M., Mishin, A. S., Yampolsky, I. V., Belousov, V. V., Chudakov, D. M., Subach, F. V., \& Lukyanov, K. A. (2009).
Green fluorescent proteins are light-induced electron donors. Nature Chemical Biology, 5(7), 459-461. https://doi.org/10.1038/ nchembio. 174

30. Subach, O. M., Patterson, G. H., Ting, L.-M., Wang, Y., Condeelis, J. S., \& Verkhusha, V. V. (2011). A photoswitchable orangeto-far-red fluorescent protein, PSmOrange. Nature Methods, 8(9), 771-777. https://doi.org/10.1038/nmeth.1664

31. Gorbachev, D. A., Petrusevich, E. F., Kabylda, A. M., Maksimov, E. G., Lukyanov, K. A., Bogdanov, A. M., \& Mishin, A. S. (2020). A general mechanism of green-to-red photoconversions of GFP. Frontiers in Molecular Biosciences, 7, 176. https://doi.org/10. 3389/fmolb.2020.00176

32. Herman, P., Holoubek, A., \& Brodska, B. (2019). Lifetime-based photoconversion of EGFP as a tool for FLIM. Biochimica et Biophysica Acta. General Subjects, 1863(1), 266-277. https://doi.org/ 10.1016/j.bbagen.2018.10.016

33. Kremers, G.-J., Hazelwood, K. L., Murphy, C. S., Davidson, M. W., \& Piston, D. W. (2009). Photoconversion in orange and red fluorescent proteins. Nature Methods, 6(5), 355-358. https://doi. org/10.1038/nmeth.1319

34. Verkhusha, V. V., Chudakov, D. M., Gurskaya, N. G., Lukyanov, S., \& Lukyanov, K. A. (2004). Common pathway for the red chromophore formation in fluorescent proteins and chromoproteins. Chemistry \& Biology, 11(6), 845-854. https://doi.org/10. 1016/j.chembiol.2004.04.007

35. Shaner, N. C., Campbell, R. E., Steinbach, P. A., Giepmans, B. N. G., Palmer, A. E., \& Tsien, R. Y. (2004). Improved monomeric red, orange and yellow fluorescent proteins derived from Discosoma sp. red fluorescent protein. Nature Biotechnology, 22(12), 1567-1572. https://doi.org/10.1038/nbt1037

36. Pletneva, N., Pletnev, S., Tikhonova, T., Popov, V., Martynov, V., \& Pletnev, V. (2006). Structure of a red fluorescent protein from Zoanthus, zRFP574, reveals a novel chromophore. Acta Crystallographica Section D, Biological Crystallography, 62(Pt 5), 527-532. https://doi.org/10.1107/S0907444906007852

37. Pakhomov, A. A., \& Martynov, V. I. (2007). Chromophore aspartate oxidation-decarboxylation in the green-to-red conversion of a fluorescent protein from Zoanthus sp. 2. Biochemistry, 46(41), 11528-11535. https://doi.org/10.1021/bi700721x

38. Pletneva, N., Pletnev, V., Tikhonova, T., Pakhomov, A. A., Popov, V., Martynov, V. I., \& Pletnev, S. (2007). Refined crystal structures of red and green fluorescent proteins from the button polyp Zoanthus. Acta Crystallographica. Section D, Biological Crystallography, 63(Pt 10), 1082-1093. https://doi.org/10.1107/S0907 444907042461

39. Kim, S. E., Hwang, K. Y., \& Nam, K. H. (2019). Spectral and structural analysis of a red fluorescent protein from Acropora digitifera. Protein Science: A Publication of the Protein Society, 28(2), 375-381. https://doi.org/10.1002/pro.3540

40. Marchant, J. S., Stutzmann, G. E., Leissring, M. A., LaFerla, F. M., \& Parker, I. (2001). Multiphoton-evoked color change of DsRed as an optical highlighter for cellular and subcellular labeling. Nature Biotechnology, 19(7), 645-649. https://doi.org/10. $1038 / 90249$

41. Snellenburg, J. J., Laptenok, S., Seger, R., Mullen, K. M., \& van Stokkum, I. H. M. (2012). Glotaran: A java-based graphical user interface for the R package TIMP. Journal of Statistical Software, Articles, 49(3), 1-22. https://doi.org/10.18637/jss.v049.i03

42. Subach, F. V., \& Verkhusha, V. V. (2012). Chromophore transformations in red fluorescent proteins. Chemical Reviews, 112(7), 4308-4327. https://doi.org/10.1021/cr2001965

43. Subach, O. M., Gundorov, I. S., Yoshimura, M., Subach, F. V., Zhang, J., Grüenwald, D., \& Verkhusha, V. V. (2008). Conversion of red fluorescent protein into a bright blue probe. Chemistry \& Biology, 15(10), 1116-1124. https://doi.org/10.1016/j.chembiol. 2008.08.006 
44. Reid, B. G., \& Flynn, G. C. (1997). Chromophore formation in green fluorescent protein. Biochemistry, 36(22), 6786-6791. https://doi.org/10.1021/bi970281w

45. Cloin, B. M. C., De Zitter, E., Salas, D., Gielen, V., Folkers, G. E., Mikhaylova, M., \& Kapitein, L. C. (2017). Efficient switching of $\mathrm{mCherry}$ fluorescence using chemical caging. Proceedings of the National Academy of Sciences of the United States of America, 114(27), 7013-7018. https://doi.org/10.1073/pnas.1617280114

46. Heppert, J. K., Dickinson, D. J., Pani, A. M., Higgins, C. D., Steward, A., Ahringer, J., \& Goldstein, B. (2016). Comparative assessment of fluorescent proteins for in vivo imaging in an animal model system. Molecular Biology of the Cell, 27(22), 3385-3394. https://doi.org/10.1091/mbc.E16-01-0063

47. Chattoraj, M., King, B. A., Bublitz, G. U., \& Boxer, S. G. (1996). Ultra-fast excited state dynamics in green fluorescent protein: Multiple states and proton transfer. Proceedings of the National Academy of Sciences of the United States of America, 93(16), 8362-8367. https://doi.org/10.1073/pnas.93.16.8362

48. Shcherbo, D., Merzlyak, E. M., Chepurnykh, T. V., Fradkov, A. F., Ermakova, G. V., Solovieva, E. A., \& Chudakov, D. M. (2007). Bright far-red fluorescent protein for whole-body imaging. Nature Methods, 4(9), 741-746. https://doi.org/10.1038/nmeth1083

49. Shcherbo, D., Murphy, C. S., Ermakova, G. V., Solovieva, E. A., Chepurnykh, T. V., Shcheglov, A. S., \& Chudakov, D. M. (2009). Far-red fluorescent tags for protein imaging in living tissues. The
Biochemical Journal, 418(3), 567-574. https://doi.org/10.1042/ BJ20081949

50. Cranfill, P. J., Sell, B. R., Baird, M. A., Allen, J. R., Lavagnino, Z., de Gruiter, H. M., \& Piston, D. W. (2016). Quantitative assessment of fluorescent proteins. Nature Methods, 13(7), 557-562. https://doi.org/10.1038/nmeth.3891

51. Shemiakina, I. I., Ermakova, G. V., Cranfill, P. J., Baird, M. A., Evans, R. A., Souslova, E. A., \& Shcherbo, D. (2012). A monomeric red fluorescent protein with low cytotoxicity. Nature Communications, 3, 1204. https://doi.org/10.1038/ncomms2208

52. Dean, K. M., Lubbeck, J. L., Binder, J. K., Schwall, L. R., Jimenez, R., \& Palmer, A. E. (2011). Analysis of red-fluorescent proteins provides insight into dark-state conversion and photodegradation. Biophysical Journal, 101(4), 961-969. https://doi.org/10. 1016/j.bpj.2011.06.055

53. Manna, P., \& Jimenez, R. (2015). Time and frequency-domain measurement of ground-state recovery times in red fluorescent proteins. The Journal of Physical Chemistry. B, 119(15), 49444954. https://doi.org/10.1021/acs.jpcb.5b00950

54. Klementieva, N. V., Pavlikov, A. I., Moiseev, A. A., Bozhanova, N. G., Mishina, N. M., Lukyanov, S. A., \& Mishin, A. S. (2017). Intrinsic blinking of red fluorescent proteins for super-resolution microscopy. Chemical Communications (Cambridge, England), 53(5), 949-951. https://doi.org/10.1039/c6cc09200d 